\title{
Identifying ground displacement trends in Bucharest using InSAR
}

\author{
(summarizing of research findings at CRMD)
}

\author{
Iuliana Armaş \\ Department of Geomorphology-Pedology-Geomatics \\ University of Bucharest, Faculty of Geography \\ Bucharest, Romania \\ iulia_armas@geo.unibuc.ro
}

Diana A. Mendes

Department of Quantitative Methods for Management and Economics

ISCTE-IUL and BRU-IUL

Lisbon, Portugal

diana.mendes@iscte.pt

\author{
Răzvan-Gabriel Popa \\ Institute of Geochemistry and Petrology \\ ETH Zürich \\ Zürich, Switzerland \\ razvan.popa@erdw.ethz.ch
}

\author{
Mihaela Gheorghe \\ Faculty of Geodesy \\ Technical University of Civil Engineering of Bucharest \\ Bucharest, Romania \\ mihaela.gheorghe@utcb.ro
}

\author{
Diana Popovici \\ Department of Geomorphology-Pedology-Geomatics \\ University of Bucharest, Faculty of Geography \\ Bucharest, Romania \\ diana_popovici@geo.unibuc.ro
}

\begin{abstract}
Some of the most popular multi-temporal interferometric techniques used in monitoring surface movements have been applied for capturing the spatial evolution patterns of Bucharest. Three sets of $C$ and $X$-band single polarized SAR images (Envisat, ERS and TSX) were processed using the PS (Persistent Scatterers) and SBAS (Small BAseline Subset) techniques. The processing results consisted of deformation maps offering an image of the line of sight displacement rates in the past. In both PS and SBAS cases, displacement rates values are of the orders of millimetres. The analysis of possible trends is focused on relevant areas with a potentially interesting historical evolution, such as former clay pits, landfill excavation sites and industrial platforms. The InSAR techniques made it possible for the first time to investigate movements over large urban areas in combination with results derived from classical methodologies (diachronic cartography, geology, geomorphology) and thorough statistical analysis. The analyses lead to a new hypothesis regarding the geological processes that affect the surface evolution patterns in Bucharest.
\end{abstract}

Keywords- Bucharest; PS InSAR; SBAS InSAR; deformation maps; ground displacement trends; transpressional system

\section{INTRODUCTION}

Captured the space-time evolution of Bucharest, was a constant research goal at the CRMD center of the University of Bucharest, Faculty of Geography (www.geodinamic.ro). Early research focused mainly on different vulnerabilities of the urban environment, and more recent issues addressed explicitly instabilities occurred from ground behavior and displacement trends based on multi-temporal InSAR methodologies (applied to ERS, ENVISAT and TerraSAR-X satellite data). The radar displacement products were validated using Global Navigation Satellite Systems (GNSS), leveling geodetic measurements, and by conventional geological and geomorphological methods. The present paper summarizes the main outcomes of our research effort, aiming to draw a more concise image of ground evolution patterns in Bucharest.

\section{MethodOLOGY}

For analyzing ground deformation in Bucharest, we processed 79 SAR images acquired by the European Space Agency (ESA) ERS -1/-2 and ENVISAT satellites and by the German Aerospace Centre (DLR) TerraSAR-X (TSX) satellite. The images covered a time span of almost 20 years, with the historical satellite data consisting of 28 ERS-1/-2 images, acquired between 1992 and 1999, 24 
ENVISAT images, acquired between 2003 and 2010 and 27 high resolution images acquired by the TSX satellite between 2011 and 2014. Because the interferometric phase is influenced by a series of factors like the signal strength, the satellite-target relative position or temporal changes in the scene or atmospheric fluctuations, these images were selected from a larger pool of SAR data by eliminating those acquired under poor weather conditions or datasets with large perpendicular.

Two of the most known InSAR techniques were used to process the data stacks: The Persistent Scatterer technique and the Small Baseline Subset technique.

The Persistent Scatterer technique was introduced by [1] to overcome some limitations of the SAR interferometry, often affected by temporal, atmospheric or geometrical decorrelation. The method is applied for ground monitoring and it is based on identifying stable reflectors in the SAR scenes of the same area. The stable reflectors are known as persistent scatterers and are represented by natural and mostly man-made objects that are usually coherent to the radar signal (buildings, infrastructure, light poles, rocks, etc). The scatterers are useful in identifying crust deformations with millimeter accuracy and to improve DEM accuracies (submeter level) over large areas. In the PS interferometric process, one master file is defined for each data stack (i.e., ERS, ENVISAT and TSX), and the interferograms are formed relative to the defined master image.

The basic principle of the SBAS technique consists in a suitable combination of multiple interferograms with corresponding small baselines [2]. Instead of a single master file, this technique generated small baseline interferograms that result from coupling SAR images with suitable temporal and spatial baseline. The resulted small baseline interferograms are implemented in a linear model where the combination of small baseline depends on the vector containing values of the unwrapped differential interferometric phase. By solving the linear model, the sampling rate of the method is increased, while phase noise is reduced and backscatter preserved by choosing a coherence threshold. Like in the case of the PS method, only coherent pixels are exploited, but in this case, the values of the coherent pixels are interpolated over larger areas using Delaunay triangulation as described by [3].

The PS processing chain has been applied to all 3 stacks of SAR images, while the SBAS was only used to process the TSX database. SARMAP's SARscape software was employed for carrying out the processing. The InSAR pairs selected for the PS algorithm were referenced to a master acquisition from 26th of June, 2012. For the SBAS algorithm, a perpendicular base of less than $500 \mathrm{~m}$ and a temporal baseline of less than 2 years were set as parameters. With these considerations we obtained a number of 26 interferograms for the PS algorithm and 178 for the SBAS algorithm. The influence of topography was removed by using an external Digital Elevation Model (DEM), a level 4 SRTM-X, available from DLR.

\section{Results AND Discussions}

\section{A. InSAR}

The results of the processing techniques consisted of deformation maps over Bucharest (Fig. 1). The techniques applied can measure very small deformations (e.g., 1 $\mathrm{mm} /$ year) allowing a synoptic view of Bucharest ground deformations. Over the urban and peri-urban areas, PS InSAR analysis resulted in approximately 30,000 PS points from ERS data, more than 60,000 for ENVISAT and almost 600,000 points for TSX. The SBAS analysis on TSX data returned over $1,000,000$ points. In each of the represented point, the algorithms determined yearly displacement rates ranging between -8 to $2 \mathrm{~mm} / \mathrm{yr}$ and displacement values from an acquisition date to another. The estimated velocity precisions were $\sim 0.3 \mathrm{~mm} / \mathrm{yr}$ for ERS and ENVISAT and $\sim 0.2 \mathrm{~mm} / \mathrm{yr}$ for TSX.

The resulted velocity values were validated by means of GNSS technology. We found a good agreement between displacement rates resulted from InSAR data and terrestrial measurements [4].

\section{B. Tested areas: Historical Clay Pits and Excavation Sites}

During an urban area's development, there is a rising demand for construction materials. The main local construction material in Bucharest was clay for bricks. Diachronic analysis of historical clay pits and excavation sites in Bucharest was based on archived cartographic materials (Table 1). In total, 67 historical pits were identified. The clay pits were located at the periphery of the city, and their surfaces would vary up to almost 20 square meters. The pits with the largest surface were: Cerchez and Tonola in the North, Vergului in the East, and Cuţarida and Ouatu in North-West. From a geomorphological point of view, most of the pits are found on the interfluve between the Dâmboviţa and Colentina Rivers; only a few of them are found in the Dâmboviţa River's corridor (Fig. 2).

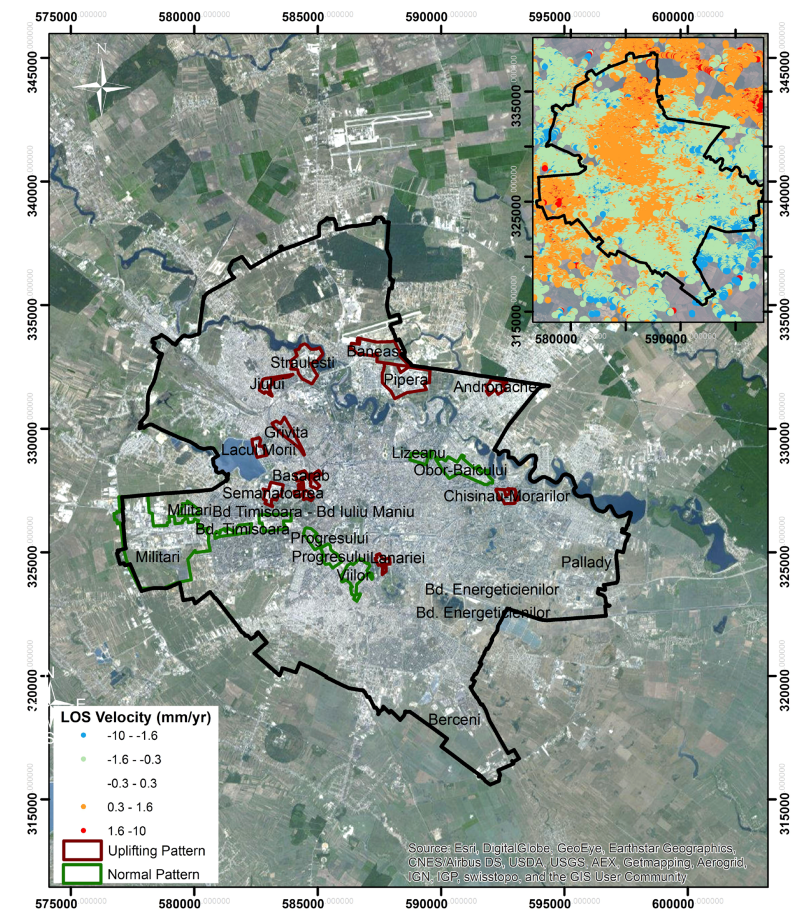

Fig. 1. Ground deformations over industrial parks. Right corner: SBAS deformation map 
TABLE 1. AREA OF EXCAVATION SITES

\begin{tabular}{|c|c|c|c|}
\hline \multirow{2}{*}{$\begin{array}{c}\text { Maps and } \\
\text { cartographic planes } \\
\text { (Year) }\end{array}$} & $\begin{array}{c}\text { Number } \\
\text { of } \\
\text { excavations }\end{array}$ & \multicolumn{2}{|c|}{ Area (ha) } \\
\cline { 3 - 4 } & 17 & 19.3 & $\begin{array}{c}\text { Maximum } \\
\text { area of an } \\
\text { excavation }\end{array}$ \\
\hline $\begin{array}{c}1854 \\
\text { (Borroczyn Plane) }\end{array}$ & 19 & 15.3 & 12.4 \\
\hline $\begin{array}{c}1871 \\
\text { (Pappasoglu Plane) }\end{array}$ & 39 & 91.2 & 2.8 \\
\hline 1914 & 3 & 5.6 & 16.8 \\
\hline 1940 & & & 3.1 \\
\hline
\end{tabular}

Between 1854 and 1871, the number of excavations increased, while their areal extent decreased. This is mainly because the number of large excavations was reduced in favour of constructing new buildings. During this period, many brick factories (e.g. Tonola, Cerchez) were established, together with small outdoor workshops; the periphery of the city contained many pits. Between 1917 and 1940, a significant decrease in this type of activity could be observed, most of the former clay pits and excavation sites being upgraded to different land use categories, and integrated into the urban landscape. After 1940, excavation sites from outside the city ceased to be marked on maps.

An uplift trend can be identified for the former excavation areas that are on the interfluves between Dâmboviţa and Colentina (Witing Street, the Ştefan Cel Mare-Dinu Vintilă Boulevards in Fig. 2).

The general uplifting trend of excavations found on interfluves aligns with the regional uplift movement (although 10 of the excavations show subsidence in the period 1992-2000, which we link to the fluctuations of the groundwater table, as explained later).

The subsidence trend was detected at a historical excavation site located around the current Bucharest Autonomous Transport Company (RATB) car shed Dudeşti, found on the Dâmboviţa terrace, as a consequence of tectonic conditions, discussed later in the paper.

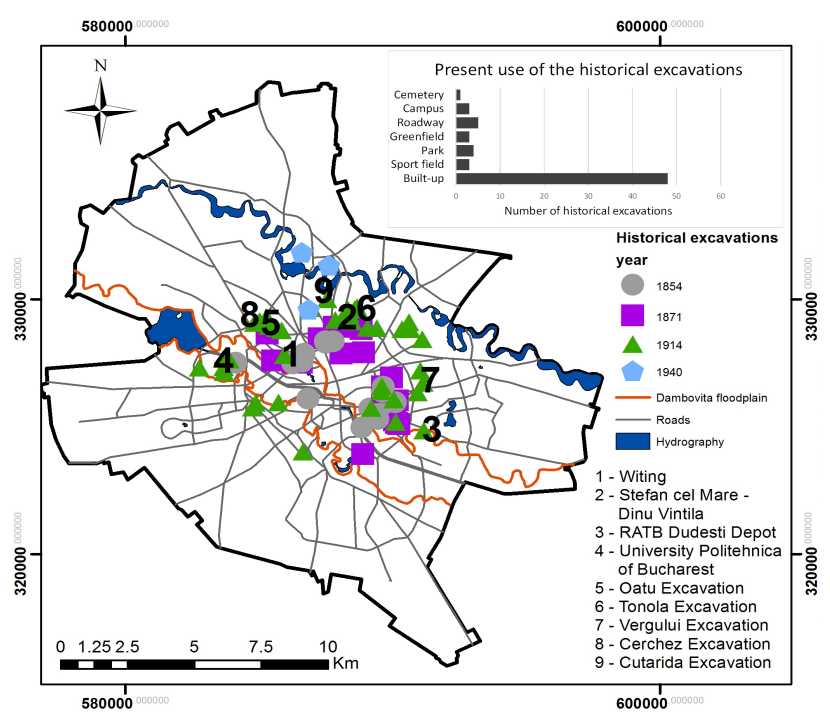

Figure 2. Historical clay pit excavations

\section{Tested areas: Industrial parks}

We concentrated our analysis on large areas that were most affected by human influence. We calculated the mean deformation rates in each period for 20 important industrial parks.

Movement trends over each of the study areas were analyzed through manual recognition and classification. The behavior of the areas, translated in movement trends, were interpreted considering geomorphologic characteristics, groundwater level changes over time, and historical evolution of the area [5]. We have discovered three main dynamic trends of the industrial areas. In this paper we will focus only on the expected (or normal) pattern with subsidence until 1999 followed by uplift after 2003, and the pattern characterized by continuous uplift. The third pattern, a subsident one, was detailed in previous research and will not be developed in this paper [5] [6]

The selection of the study areas was made especially considering criteria of human impact, in order to identify the long-term dynamic pattern, with natural causes, disturbed by medium-term human impact. The analysis of the industrial areas described in a former study [5] represented a basis for a more detailed investigation in a paper published in Scientific Reports [6]. Different industrial parks in Bucharest represent suitable test sites for identifying long-term dynamic patterns through comparative analysis with their neighboring areas. The main hypothesis of our research was that areas affected by human impact are recovering after shutting down most of the industry in Bucharest and are tending towards the normal long-term trend of ground evolution. The long-term trends are emphasized by contrast with the short- or medium-term trends of the human impacted areas within the city. Complex statistical analyses were carried out and the results were presented on different occasions [4] [5] [6].

\section{Ground displacement patterns}

After applying statistical and numerical tests, we have identified a persistent long memory behavior in each of the analyzed displacement time series.

The long-term behavior describes three quasi-parallel NW-SE uplift domains separated by 2 subsidence corridors. The main uplift area corresponds to the interfluve between Dâmbovița and Colentina rivers. Interestingly, this area hosts most of the buildings that are catalogued as class I, II and III of seismic risk, and most of the buildings that have collapsed during the 1977 earthquake.

Investigating the ground deformations caused by the identified uplift is hindered by the geological substrate of Bucharest, made of unconsolidated or plastic rocks that do not easily retain visible deformation or movement markers. To overcome this issue, our working hypothesis considered that taller buildings with deep foundation are better coupled with the geological substrate and might react similarly. The buildings however, have the ability to record movement or deformation markers via fractures or fissures in their structures.

A classical geological methodology has been applied in an unconventional way: the azimuth of the discontinuities that could be traced in more than 1 structural plane of the 
investigated buildings has been measured with the geological compass. The distribution of fissures in the man-made structures are indicated of dissociated stresses induced by a NW-SE strain field. Thus, the connection between the NW-SE uplift domain and the compromising of the buildings structures is a potential conclusion. Bucharest is located just $40 \mathrm{~km}$ west of the Intra-Moesian Fault Line, and it seems likely that the city was built on a peripheral section of this fault system. Differentiated movement (different velocities) on the main faults or on the secondary adjustment faults can create transpressional fields that result in shortening and uplift.

\section{CONCLUSIONS}

The main challenges we had to face in our attempt to identify ground displacement trends in Bucharest were a low backscattered radar signal in the absence of a validated neotectonic model, and the lack of permanent GNSS stations within the city to test our InSAR results. We overcome these aspects by selecting only highly reliable imagery from a larger pool of data, and installing throughout the city centering systems at ground level for GNSS receivers. Therefore, we were able to obtain and validate deformation maps that describe Bucharest's ground surface movement patterns. We then carried out complex statistical analysis on the resulted data that pointed out to a nonlinear periodic process caused by possible attractors, possible explained by an active transpressional system that Bucharest is built on.

\section{ACKNOWLEDGMENT}

This research was financed by grant 78/29.11.2013 of the Romanian Space Agency. ERS-1/-2 and ENVISAT data were provided by ESA under the ESA AO CAT-1 proposal (i.e., Project 12793), PI's M. Necșoiu and I. Armaș. TSX data were provided via DLR Proposal LAN 1444, PI's I. Armaş and M. Necşoiu. We would like to thank Dr. Eng. Marius Necșoiu for support in processing InSAR data.

\section{REFERENCES}

[1] A. Ferretti, C. Prati, and F. Rocca, "Permanent scatterers in SAR interferometry," IEEE Transactions on geoscience and remote sensing, vol. 39, no. 1, pp. 8-20, 2001.

[2] P. Berardino, G. Fornaro, R. Lanari, and E. Sansosti, "A new algorithm for surface deformation monitoring based on small baseline differential SAR interferograms”, IEEE Transactions on Geoscience and Remote Sensing, vol. 40, no. 11, pp. 2375-2383, 2002.

[3] M. Costantini, and P.A. Rosen, “A generalized phase unwrapping approach for sparse data”, Geoscience and Remote Sensing Symposium. IGARSS'99 Proceedings. IEEE 1999 International, vol. 1, pp. 267-269, 1999.

[4] I. Armaș, M. Gheorghe, A.M. Lendvai, P.D. Dumitru, O. Bădescu, and A. Călin, "InSAR validation based on GNSS measurements in Bucharest,” International Journal of Remote Sensing, vol. 37, no. 23, pp. 5565-5580, 2016.

[5] I. Armaș, M. Necsoiu, D.A. Mendes, M. Gheorghe, D. Gheorghe, "Ground Displacement Trends in an Urban Environment Using Multi-Temporal InSAR Analysis and Two Decades of MultiSensor Satellite-Based SAR Imagery,” In Proceedings of ESA 9th International Workshop Fringe 2015 Advances in the Science and Applications of SAR Interferometry and Sentinel-1 InSAR Workshop, ESA SP, Vol. 731, May 2015.

[6] I. Armaş, D. Mendes, R.-G. Popa, M. Gheorghe, D. Popovici, "Long-term ground deformation patterns of Bucharest using multitemporal InSAR and multivariate dynamic analyses: a possible transpressional system?”, Sci. Rep. 7, 43762, 2017. doi: 10.1038/srep43762 